\title{
Study and Simulation of Deformation Mechanics Modeling of Flexible Workpiece Processing by Rayleigh-Ritz Method
}

\author{
Yaohua Deng, ${ }^{1}$ Sicheng Chen, ${ }^{1}$ Qiwen Lu, ${ }^{2}$ Jiayuan Chen, ${ }^{1}$ Qiaofen Zhang, \\ Liming $\mathrm{Wu}{ }^{1}{ }^{1}$ Jiandong Shi, ${ }^{1}$ and Xiali Liu ${ }^{1}$ \\ ${ }^{1}$ School of Information Engineering, Guangdong University of Technology, No. 100 Waihuanxi Road, \\ Guangzhou Higher Education Mega Center, Mail Box B65, Guangdong 510006, China \\ ${ }^{2}$ HSBC Software Development (Guangdong) Limited, Guangzhou, Guangdong, China \\ Correspondence should be addressed to Yaohua Deng; dengyaohua@gdut.edu.cn
}

Received 8 May 2014; Accepted 17 June 2014

Academic Editor: Yoshinori Hayafuji

Copyright (C) 2015 Yaohua Deng et al. This is an open access article distributed under the Creative Commons Attribution License, which permits unrestricted use, distribution, and reproduction in any medium, provided the original work is properly cited.

\begin{abstract}
This paper discusses the calculation problems of bending deformation of FWP processing. Take three axis CNC machining as an example, to establish mechanics model of flexible workpiece processing process. The flexible workpiece balance equation is a two-dimensional partial differential equation, to solve the problem of flexible workpiece bending deformation using Rayleigh-Ritz method and designing the test function of bending deformation of flexible workpiece. By satisfying the minimum potential energy condition of FWP processing to work out the approximate solution of bending deformation of flexible workpiece, find out the relationship between material properties of flexible piece, acting force $F_{z}$, and deformation value. Finally, the rectangle flexible workpiece which is made up of polyurethane sponge is selected as an experiment subject. The results show that the average relative deviation between theoretical value and observed value is only $5.51 \%$. It is proved that the bending deformation test function satisfies the actual deformation calculation requirements.
\end{abstract}

\section{Introduction}

The basic material of FWP (flexible workpiece path, FWP) processing workpiece includes elastic thin plate, textile fabric, and polyurethane sponge. The constitutive model of those materials showing physical nonlinear or geometry nonlinear, the macroscopic showing low rigidity strength, small elastic modulus, and soft mechanics performance [1-3]. The FWP processing workpiece will generate deformation of bending and stretching due to the effect of processing forces; it is led to processing path deviation from the original setting path $[4,5]$. There are two type of bending deformation calculation method, one is accurate solution including Navier solution, Levy solution and ZhangFufan solution, another one is approximate solution including Rayleigh-Ritz solution and Galerkin solution. Navier solution method get the Navier solution by double sine series, convergence faster when deflection calculation, but slower when flexural moment and shear force calculation, especially under concentrated force. The flexural moment under concentrated force is not convergence; this method is only applied to four-side simply supported thin plate; Levy solution can solve the bending of four-side simply supported rectangular plate subject to various lateral loads, bending problem of distributed torque or subsidence and corner occurred subsidence, and so forth; this method is not applied to the occasion of support saddle subsidence. Each spot has established deflection value along the generalized simply supported side in the ZhangFufan method. After solving one-side or two side simply supported limit, adding four side simply supported rectangular plate limit, to get the bending solution, then solve the problem of free-side rectangular plate bending. It is suitable for the condition of rectangular plates bending with free edge. The Galerkin method is established by virtual displacement principle, under the boundary condition approximately satisfying the differential equation of rectangular plate bending, but does not need to calculate the plate deformation energy; it 


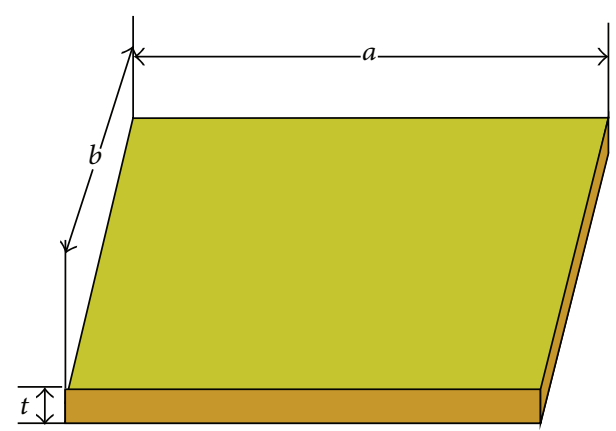

FIGURE 1: FWP processing rectangular flexible part geometric model.

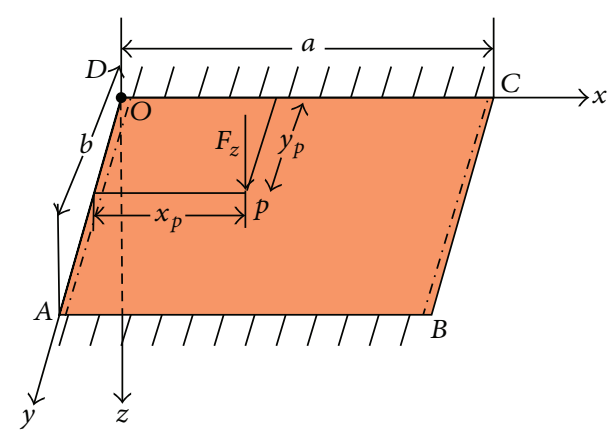

FIGURE 2: Flexible part processing mechanics model.

is more suitable for the condition of thin plate little bending deformation calculation compared with Rayleigh-Ritz solution. First, analysis the flexible workpiece mechanical model of FWP processing process, then derivation flexible workpiece bending equation $[6-8]$.

\section{The Flexible Pieces Processing Process Mechanical Model}

Figure 1 is FWP processing rectangular flexible workpiece geomectric model. In Figure 1, set the length, width, and height of flexible workpiece as $a, b$, and $c$, respectively. The flexible part clamps on the level objective table. During the path processing process, the concentrated stress of flexible workpiece is mainly $F_{z}$ which is on the direction $Z$ of the action spot $p\left(x_{p}, y_{p}\right)$. Figure 2 is the FWP simplified mechanical model.

Flexible workpiece under $F_{z}$ force, the stress vector is not perpendicular to the $x-y$ plane. Under the point, microbody $d x d y d z$ should be constituted with normal stress and shear stress (see the microbody stress diagram of point $p$ in Figure 3). Under the force of normal stress and shear stress, the microbody $d x d y d z$ produce different degree bending and shear deformation, so the flexible workpiece showing irregular behavior on the $x-y$ plane. Considering the flexible part is clamped on the 2-dimensional plane stage, generally $t / b \in[0.01,0.167]$, the external force is in the role of the middle plane of the flexible part. All flexible workpiece bending deformations have small concave curve characteristics.
Shear deformation compared with bending deformation is negligible. At this time, the flexible part's balance equation belongs to two-dimensional partial differential equation. In view of Rayleigh-Ritz solution (RRS), supposing bending displacement function could not be satisfied with the boundary conditions of force, the structure of the displacement function also is relatively easy. Below, the Rayleigh-Ritz solution is used to discuss the flexible part bending deformation. By satisfying the minimum potential energy condition of FWP flexible piece processing process to work out the approximate solution of flexible piece bending deformation, find out the relationship between material properties of flexible piece, acting force $F_{z}$, and deformation [9].

\section{Flexible Part Bending Deformation RRS Equation}

3.1. Flexible Part Bending Deformation RRS Solving Assumption. Based on Figure 3, supposing the microbody is deformed, it is the three-component product $u, v$, and $w$ of the displacement vector that is in the coordinates $x, y$, and $z$. Flexible workpiece bending deformation solution by RRS method [10].

(1) The normal strain $\varepsilon_{z}$ on center plane is extremely small. Consider $\varepsilon_{z} \approx 0$; then, according to $\varepsilon_{z}=$ $\partial w / \partial z=0, \Rightarrow w=w(x, y)$. That is any point on the flexible part bending deformation $w$ is $x, y$ 's function. It is related to coordinates.

(2) The shear strain $\gamma_{z x} \approx 0, \gamma_{z y} \approx 0$, so

$$
\begin{gathered}
\gamma_{z x}=\frac{\partial w}{\partial x}+\frac{\partial u}{\partial z}=0, \quad \gamma_{z y} \frac{\partial w}{\partial y}+\frac{\partial v}{\partial z}=0 \\
\Longrightarrow \frac{\partial u}{\partial z}=-\frac{\partial w}{\partial x}, \quad \frac{\partial v}{\partial z}=-\frac{\partial w}{\partial y} .
\end{gathered}
$$

(3) Flexible workpiece bending deformations, each point in the middle plane did not produce the strain that is parallel to this plane.

(4) In the stable state, flexible part minimum potential energy condition is equivalent to flexible part bending surface differential equation.

3.2. The RRS Equation Solution Process of Flexible Part Bending Deformation. Based on the assumption above, the flexible part's elastic modulus is $E$ and Poisson ratio is $u$; then, the flexural rigidity is $D=E t^{3} / 12\left(1-u^{3}\right)$, microbin is the sum of strain energy $U$ and external force potential. Total potential energy function [11] is

$$
\begin{aligned}
\prod & =U+V=\int_{0}^{a} \int_{0}^{b}\left[\frac{D}{2}\left(\frac{\partial^{2} w}{\partial x^{2}}+\frac{\partial^{2} w}{\partial y^{2}}\right)^{2}-F_{z} w\right] d x d y \\
& =\frac{D}{2} \int_{0}^{a} \int_{0}^{b}\left(\frac{\partial^{2} w}{\partial x^{2}}\right)^{2} d x d y
\end{aligned}
$$




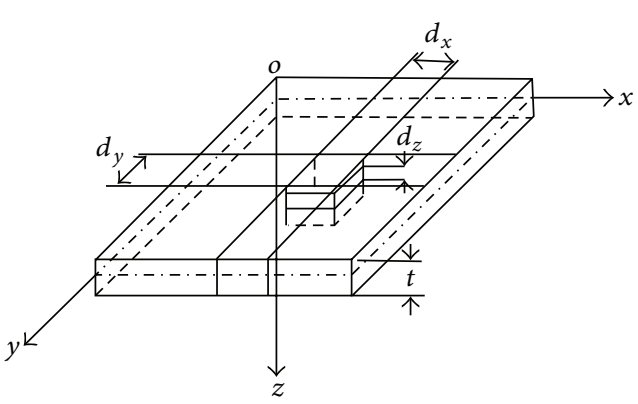

(a)

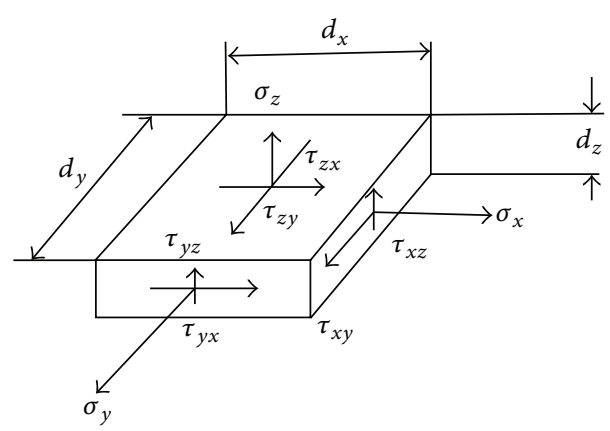

(b)

Figure 3: Microbody stress analysis diagram under point $p$.

$$
\begin{aligned}
& +\frac{D}{2} \int_{0}^{a} \int_{0}^{b}\left(\frac{\partial^{2} w}{\partial y^{2}}\right)^{2} d x d y \\
& +D \int_{0}^{a} \int_{0}^{b} \frac{\partial^{2} w}{\partial X^{2}} \frac{\partial^{2} w}{\partial y^{2}} d x d y-\int_{0}^{a} \int_{0}^{b} F_{z} w d x d y
\end{aligned}
$$

Set

$$
\begin{aligned}
& U_{1}=\frac{D}{2} \int_{0}^{a} \int_{0}^{b}\left(\frac{\partial^{2} w}{\partial x^{2}}\right)^{2} d x d y \\
& U_{2}=\frac{D}{2} \int_{0}^{a} \int_{0}^{b}\left(\frac{\partial^{2} w}{\partial y^{2}}\right)^{2} d x d y, \\
& U_{3}=D \int_{0}^{a} \int_{0}^{b} \frac{\partial^{2} w}{\partial X^{2}} \frac{\partial^{2} w}{\partial y^{2}} d x d y, \\
& V=\int_{0}^{a} \int_{0}^{b} F_{z} w d x d y,
\end{aligned}
$$

then,

$$
\prod=U_{1}+U_{2}+U_{3}-V .
$$

Set the bending deformation test function with the force of $F_{z}$ as follows:

$$
w=A_{1} y \sin \frac{\pi x}{a}+A_{2} \sin \frac{\pi y}{b}+A_{3} \sin \frac{\pi x}{a} \sin \frac{\pi y}{b},
$$

where $A_{1}, A_{2}$, and $A_{3}$ are pending constant and $a$ and $b$ are the length and width of the flexible part.

Figure 2 is the flexible workpiece geometric model. If the flexible part's fixed edge is $A B, C D$, then the winding degree and surface slope of $A B, C D$ are 0 . And along the simple edge $A B, C D$, the moment and the strain are 0 on the direction $x$ as follows:

$$
\begin{gathered}
(w)_{y=0 \text { or } b}=\left(\frac{\partial w}{\partial y}\right)_{y=0 \text { or } b}=0, \\
\left(\frac{\partial^{2} w}{\partial x^{2}}\right)_{x=0 \text { or } a}=\left(\frac{\partial^{2} w}{\partial x^{2}}+u \frac{\partial^{2} w}{\partial y^{2}}\right)_{x=0 \text { or } a}=0 .
\end{gathered}
$$

Basing on (5), it shows

$$
\left\{\begin{array}{l}
\frac{\partial w}{\partial y}=A_{1} \sin \frac{\pi x}{a}+A_{2} \frac{\pi}{b} \cos \frac{\pi y}{b}+A_{3} \frac{\pi}{b} \sin \frac{\pi x}{a} \cos \frac{\pi y}{b} \\
\left(\frac{\partial w}{\partial y}\right)_{y=0}=0
\end{array}\right.
$$$$
\Longrightarrow A_{1}+\frac{4}{b} A_{2}+\frac{\pi}{b} A_{3}=0 .
$$

Similarly, work out by (5) as follows:

$$
\begin{aligned}
& \left\{\begin{array}{l}
\frac{\partial^{2} w}{\partial x^{2}}=-A_{1} \frac{\pi^{2}}{a^{2}} y \sin \frac{\pi x}{a}-A_{3} \frac{\pi^{2}}{a^{2}} \sin \frac{\pi x}{a} \sin \frac{\pi y}{b}=0, \\
\frac{\partial^{2} w}{\partial x^{2}}{ }_{x=0}=0
\end{array}\right. \\
& \Longrightarrow A_{1}=-\frac{4}{\pi b} A_{3} .
\end{aligned}
$$

Uniting (6), (7), and (8), work out the testing function of bending deformation $w$ as

$$
\begin{gathered}
w=A_{3}\left[-\frac{4}{\pi b} y \sin \frac{\pi x}{a}+\left(\frac{1}{\pi}-\frac{\pi}{4}\right) \sin \frac{\pi y}{b}\right. \\
\left.+\sin \frac{\pi x}{a} \sin \frac{\pi y}{b}\right] .
\end{gathered}
$$

According to (9) and (4),

$$
\begin{aligned}
U_{1}=\frac{D}{2} \int_{0}^{a} \int_{0}^{b}\left(\frac{4 A_{3} \pi}{a^{2} b} y \sin \frac{\pi x}{a}-\frac{A_{3} \pi}{a^{2}} \sin \frac{\pi x}{a} \sin \frac{\pi y}{b}\right)^{2} d x d y \\
\Longrightarrow U_{1}=\frac{4 D A_{3}^{2} \pi^{2} b}{3 a^{3}}+\frac{D A_{3}^{2} \pi^{4} b}{8 a^{3}}-\frac{2 D A_{3}^{2} \pi^{2} b}{a^{3}} \\
=\frac{D A_{3}^{2} \pi^{2} b}{a^{3}}\left(\frac{1}{3}+\frac{1}{8} \pi^{2}\right) .
\end{aligned}
$$




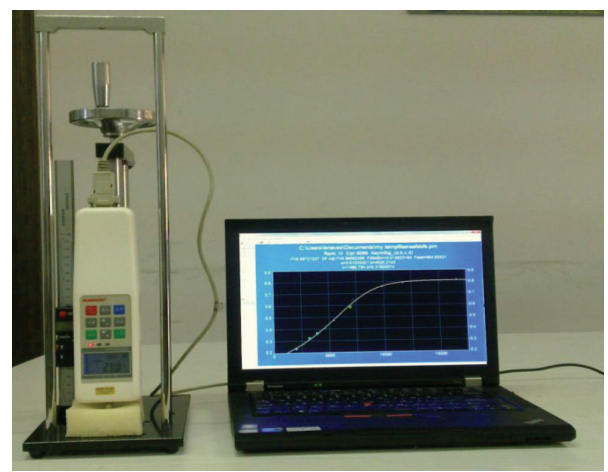

(a)

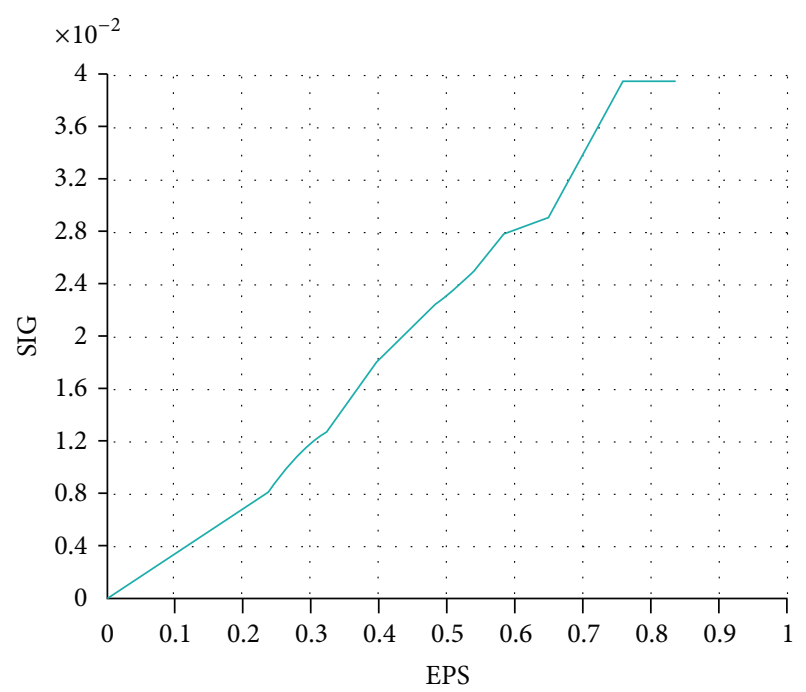

(b)

FIgURE 4: Material stress and strain characteristic curve. (a) Stress and strain test devices. (b) The stress-strain characteristic curve.

Similarly,

$$
\begin{aligned}
U_{3}= & D \int_{0}^{a} \int_{0}^{b}\left(\frac{4 A_{3}}{\pi b} y \cdot \frac{\pi^{2}}{a^{2}} \sin \frac{\pi x}{a}-\frac{A_{3} \pi}{a^{2}} \sin \frac{\pi x}{a} \sin \frac{\pi y}{b}\right) \\
& \cdot\left(-\frac{A_{3} \pi^{2}}{b^{2}}\left(\frac{1}{\pi}-\frac{\pi}{4}\right)\right) \\
& \cdot \sin \frac{\pi y}{b}-\frac{A_{3} \pi^{2}}{b^{2}} \sin \frac{\pi x}{a} \sin \frac{\pi y}{b} \\
= & \left(\frac{8}{b}+\pi^{2}\right) D A_{3}^{2} .
\end{aligned}
$$

Similarly, we can get

$$
U_{2}=\frac{D \pi^{3} A_{3}^{2} a}{b^{3}}\left(\frac{1}{4}-\frac{\pi^{2}}{4}+\frac{1}{\pi}-\frac{\pi}{8}\right) .
$$

Uniting (10), (11), and (12), we can get total strain energy $U$ as

$$
\begin{aligned}
U= & \frac{D b \pi^{2}}{a^{3}} A_{3}^{2}\left(\frac{1}{3}+\frac{1}{8} \pi^{2}\right) \\
& +\frac{D a \pi^{3}}{b^{3}} A_{3}^{2}\left(\frac{1}{4}-\frac{\pi^{2}}{4}+\frac{1}{\pi}-\frac{\pi}{8}\right)+\left(\frac{8}{b}+\pi^{2}\right) D A_{3}^{2} .
\end{aligned}
$$

Generally,

$$
\begin{aligned}
V=\int_{0}^{a} \int_{0}^{b} F_{z} A_{3}[ & -\frac{4}{\pi b} y \sin \frac{\pi x}{a}+\left(\frac{1}{\pi}-\frac{\pi}{4}\right) \sin \frac{\pi y}{b} \\
& \left.+\sin \frac{\pi x}{a} \sin \frac{\pi y}{b}\right] d x d y \\
= & 2 a b F_{z} A_{3}\left(-\frac{1}{\pi}-\frac{\pi}{4}+2\right) .
\end{aligned}
$$

By the minimum total potential energy principle, when $F_{z}$ forces on $x=x_{p}, y=y_{p}$, real displacement makes the system total potential energy take minimum value. Total potential energy $\prod$ make partial derivative to coefficient $A_{3}$, the result is zero [12] as

$$
\begin{aligned}
\frac{\partial \prod}{\partial A_{3}}=\frac{\partial(U-V)}{\partial A_{3}}=\frac{\partial U}{\partial A_{3}}-\frac{\partial V}{\partial A_{3}}=0 \\
\Longrightarrow \frac{\partial U}{\partial A_{3}}=\frac{\partial V}{\partial A_{3}} \\
\Longrightarrow A_{3}=2 a b\left(-\frac{1}{\pi}-\frac{\pi}{4}+2\right) F_{z} \\
\times\left(\left[\frac{b \pi^{2}}{a^{3}}\left(\frac{1}{3}+\frac{1}{8} \pi^{2}\right)+\frac{a \pi^{3}}{b^{3}}\left(\frac{1}{4}-\frac{\pi^{2}}{4}+\frac{1}{\pi}-\frac{\pi}{8}\right)\right.\right. \\
\left.\left.\quad+\left(\frac{8}{b}+\pi^{2}\right)\right] D\right)^{-1} .
\end{aligned}
$$

So, the bending deformation calculation expression forces on $x_{p}, y_{p}$ are

$$
\begin{aligned}
& w\left(x_{p}, y_{p}\right) \\
& =2 a b\left(-\frac{1}{\pi}-\frac{\pi}{4}+2\right) F_{z} \\
& \quad \times\left(\left[\frac{b \pi^{2}}{a^{3}}\left(\frac{1}{3}+\frac{1}{8} \pi^{2}\right)+\frac{a \pi^{3}}{b^{3}}\left(\frac{1}{4}-\frac{\pi^{2}}{4}+\frac{1}{\pi}-\frac{\pi}{8}\right)\right.\right. \\
& \left.\left.\quad+\left(\frac{8}{b}+\pi^{2}\right)\right] D\right)^{-1}
\end{aligned}
$$




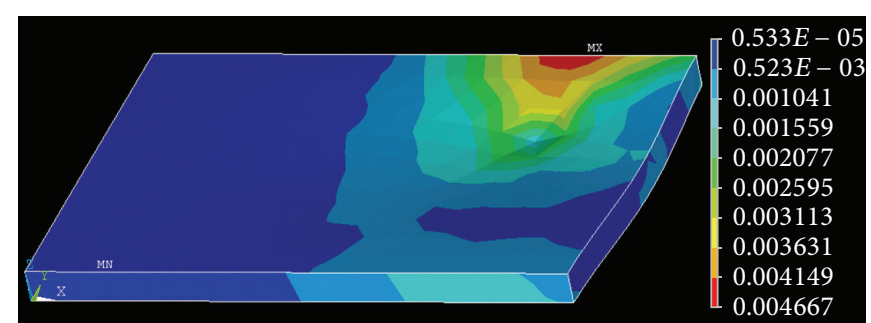

Diagram of stress distribute cloud

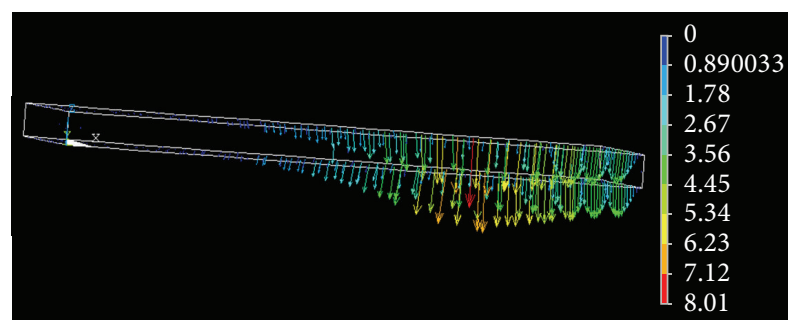

Diagram of deformation displacement vector

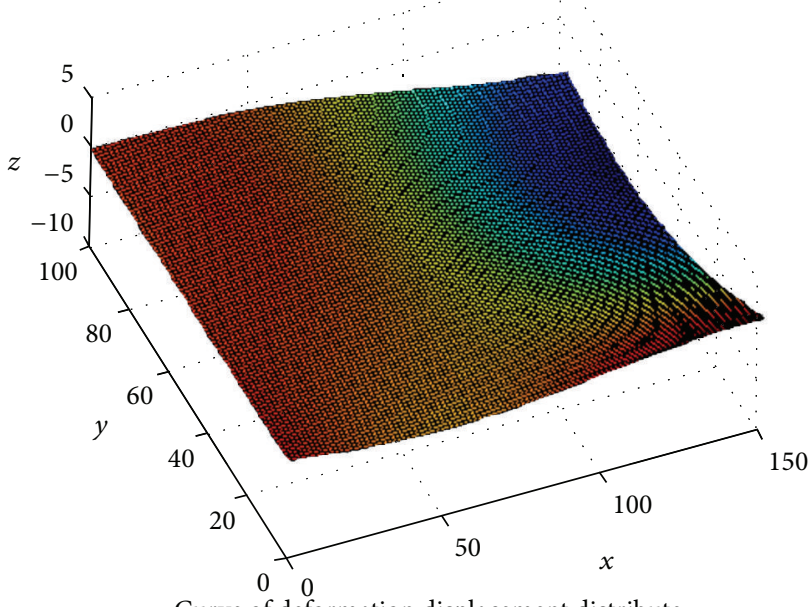

Curve of deformation displacement distribute

(a) $l=112.5, k=75, w=-1.36$

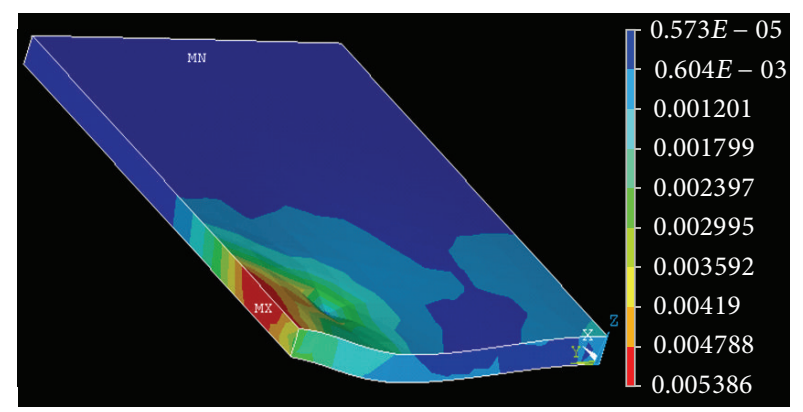

Diagram of stress distribute cloud

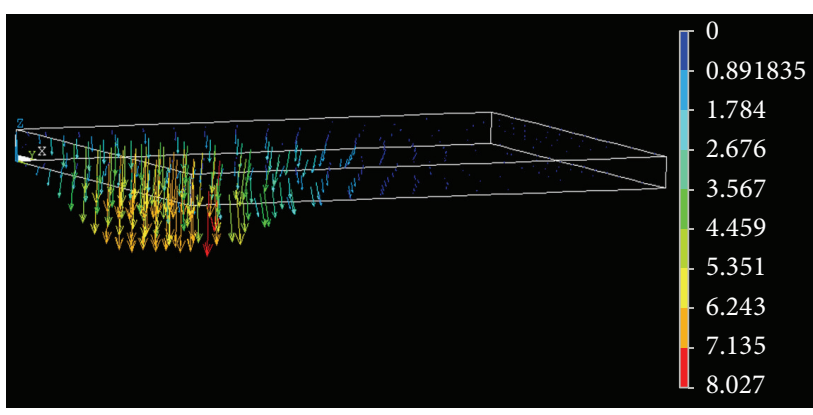

Diagram of deformation displacement vector

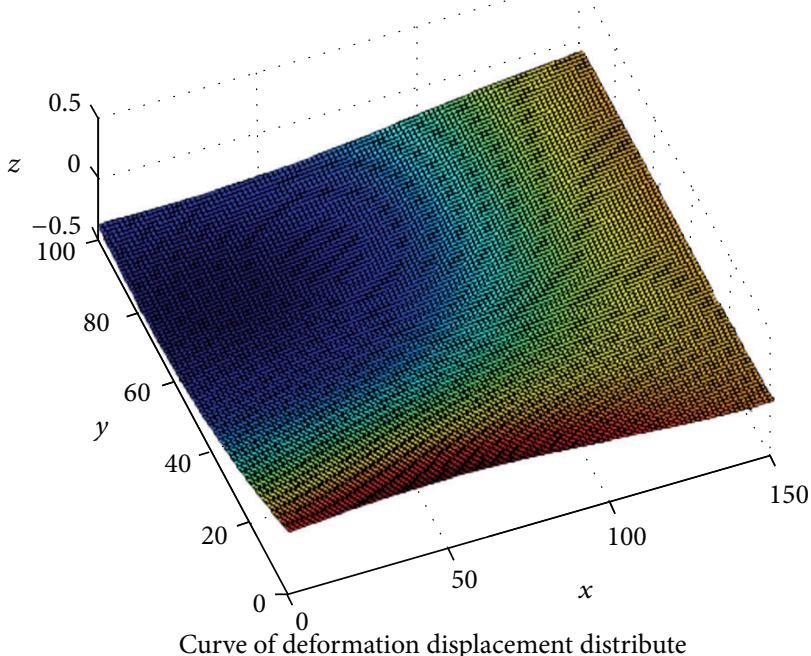

(b) $l=37.5, k=75, w=-0.38$

FIGURE 5: Processing workpiece asymmetric bending deformation stress and displacement distribute diagram. 
TABLE 1: The comparison between theoretical value and measured value of flexible part deformation.

\begin{tabular}{lcccc}
\hline Serial number & $X-Y$ plane coordinates $(\mathrm{mm})$ & Theoretical value $(\mathrm{mm})$ & Measured $(\mathrm{mm})$ & Relative deviation $(\%)$ \\
\hline 1 & $(112.5,75)$ & 3.918 & 4.242 & 7.63 \\
2 & $(37.5,75)$ & 3.916 & 4.135 & 5.29 \\
3 & $(37.5,25)$ & 3.973 & 3.767 & 5.46 \\
4 & $(112.5,25)$ & 3.262 & 3.211 & 7.58 \\
5 & $(75,50)$ & 7.508 & 7.207 & 4.17 \\
6 & $(0,50)$ & 2.795 & 2.624 & 6.51 \\
7 & $(150,50)$ & 3.815 & 4.142 & 7.89 \\
\hline \multicolumn{2}{c}{ Average } & & & 5.51 \\
\hline
\end{tabular}

$$
\begin{aligned}
& \times\left[-\frac{4}{\pi b} y_{p} \sin \frac{\pi x_{p}}{a}+\left(\frac{1}{\pi}-\frac{\pi}{4}\right) \sin \frac{\pi y_{p}}{b}\right. \\
& \left.+\sin \frac{\pi x_{p}}{a} \sin \frac{\pi y_{p}}{b}\right] \\
& =\left(F_{z}, E, t, a, b, x_{p}, y_{p}\right) .
\end{aligned}
$$

The bending deformation $w\left(x_{p}, y_{p}\right)$ is the integrating force by acting force $F_{z}$, material bending stiffness $D$ (work out by elastic modulus $E$ of flexible workpiece and its thickness $t$ ), length $a$ and width $b$ of flexible workpiece, and the function of position $p\left(x_{p}, y_{p}\right)$.

\section{Simulation Experiment}

Take flexible rectangular workpiece processing deformation as an example, the material of workpiece is polyurethane sponge, elastic modulus $E=0.2561 \mathrm{Mpa}$, Poisson coefficient $\mu=0.25$, length $a=150 \mathrm{~mm}$, width $b=100 \mathrm{~mm}$, thickness $h=8 \mathrm{~mm}$, the stress-strain characteristic curve is shown in Figure 4. Choose the point of rectangle workpiece diagonal line as loading point, concentrated load $Q=8 \mathrm{~N}$. The deformation mechanics simulation and theoretical results are shown in Figure 5. The $x$ and $y$ axis of bending displacement distribution curve is respectively represent the length and width of workpiece, the $z$ axis is represent the bending deflection $w$. All the units are mm [13].

Based on the above, measure seven points on flexible part surface in Figure 5(a). Use the digital pressure gauge with micrometer measures point by point at the same time. Finally, Table 1 shows the results of the comparison between theoretical value and measured value.

Results show that the calculated result of the average of the relative deviation is only $6.85 \%$. It shows that, according to Formula (5) given test function, we can compute the flexible part bending deformation.

\section{Conclusions}

(1) According to the actual condition of FWP processing, setting bending mechanical model and bending deflection test function under concentrated load, to derivative approximate theoretical solution of flexible workpiece processing deformation.
(2) The RRS solution hypothesis bending displacement function meets the boundary conditions of force; the structure of the displacement function also is relatively easy. Example calculation shows that setting up the FWP processing concentrated load test function by RRS is feasible.

(3) From the bending deformation function $w\left(x_{p}, y_{p}\right)$, $w$ will influence by acting force $F_{z}$, bending stiffness $D$, length $a$ and width $b$ of flexible workpiece, and force position $p\left(x_{p}, y_{p}\right)$; they are the effect factors of FWP machining deformation. It provides theory basis to the prediction modeling of deformation compensation control.

\section{Conflict of Interests}

The authors declare that there is no conflict of interests regarding the publication of this paper.

\section{Acknowledgments}

This work is partially supported by Natural Science Foundation of Guangdong Province, China (no. S2013010013288), National Natural Science Foundation of China (no. 51205069), and Combination Project of IndustryUniversity Research of Foshan Shunde of Guangdong Province, China (no. 2013CXY02). Thanks are due for the help.

\section{References}

[1] D. Yao-Hua, H. Wei, L. Qing-Fu, and W. Li-Ming, "Regression calculation model of flexible material processing deformation based on distributed sensors measurement," Advances in Information Sciences and Service Sciences, vol. 3, no. 11, pp. 208-212, 2011.

[2] D. Yaohua and L. Guixiong, "ATS-FNN-based modeling and simulation for compensation prediction of FWP machining deformation," Journal of South China University of Technology (Natural Science Edition), vol. 40, no. 3, pp. 146-151, 2012.

[3] Z. Mei, Y. Wang, and Y. Fan, "Researching and simulating deformation of aircraft structure part in NC machining," Acta Aeronautica et Astronautica Sinica, vol. 26, no. 2, pp. 234-239, 2005. 
[4] W. Yunqiao, M. Zhongyi, and F. Yuqing, "Research on deformation in NC machining of aerospace thin-walled structure part," Machinery Manufacturing Engineer, vol. 1, pp. 31-33, 2005.

[5] Z. Wang, N. He, K. Wu et al., "Analysis and control approach for machining deflection of thin-walled workpiece," China Mechanical Engineering, vol. 13, no. 2, pp. 114-117, 2002.

[6] W. Kai, H. Ning, and L. Wenhe, "Selection of cutting parameters in high speed milling of thin-walled structure components based on the machining errors control," Mechanical Science and Technology, vol. 24, no. 7, pp. 788-791, 2005.

[7] Y. Deng, S. Chen, and J. Chen, "Deformation-compensated modeling of flexible material processing based on T-S fuzzy neural network and fuzzy clustering," Journal of Vibroengineering, vol. 16, no. 3, pp. 1455-1463, 2014.

[8] C. Li, Y. Zhang, and J. Xu, "Numerical simulation of draping and buckling of woven fabric," Journal of Tianjin University Science and Technology, vol. 40, no. 1, pp. 46-50, 2007.

[9] S. F. Chen, J. L. Hu, and J. G. Teng, "A finite-volume method for contact drape simulation of woven fabrics and garments," Finite Elements in Analysis and Design, vol. 37, no. 6-7, pp. 513531, 2001.

[10] A. Demiroz, "Prediction of large deformation behavior of fabric using Galerkin finite element method," Textile Research Journal, vol. 75, no. 9, pp. 662-669, 2005.

[11] S. Pengcheng and H. Peixiang, "The developments of spline finite element method in computational mechanics," Advances in Mechanics, vol. 30, no. 2, pp. 191-199, 2000.

[12] S. Gao and C. Zhou, "Research on differentiation of the cubic spline interpolation," Science Technology and Engineering, vol. 8, no. 11, pp. 2948-2950, 2009.

[13] Y. Deng, Q. Lu, J. Chen, S. Chen, L. Wu, and L. Tang, "Study on the extraction method of deformation influence factors of flexible material processing based on information entropy," Advances in Mechanical Engineering, vol. 2014, Article ID 547947, 8 pages, 2014. 


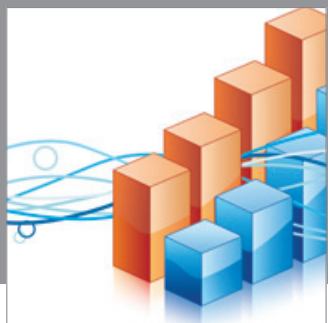

Advances in

Operations Research

mansans

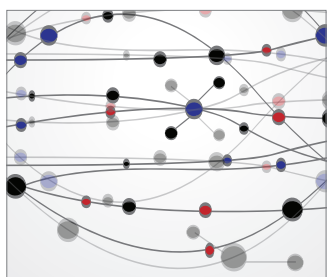

The Scientific World Journal
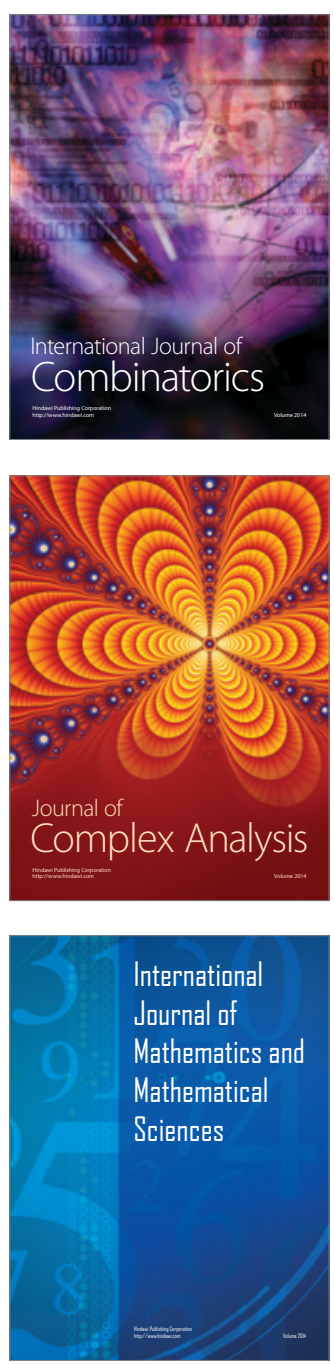
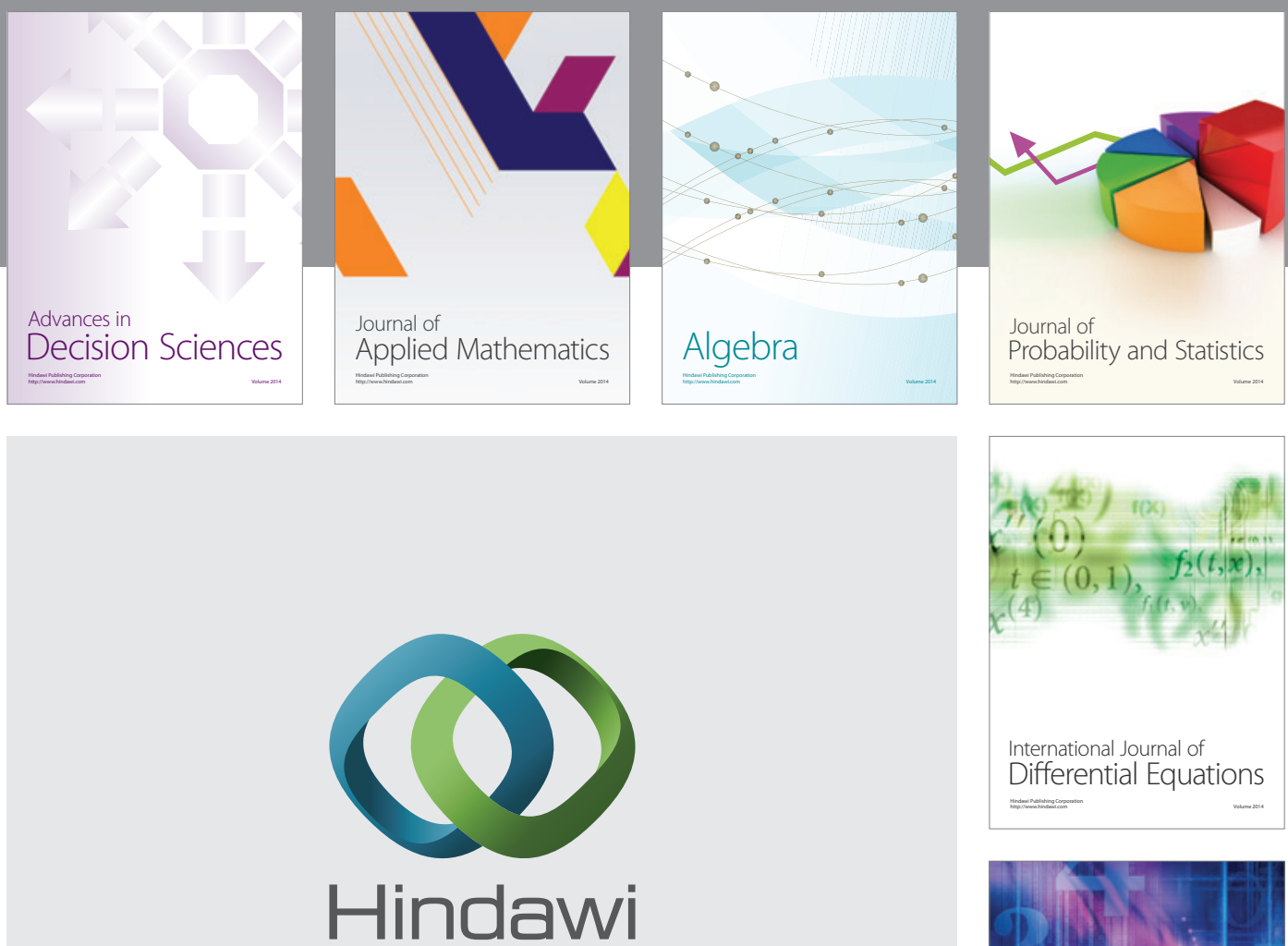

Submit your manuscripts at http://www.hindawi.com
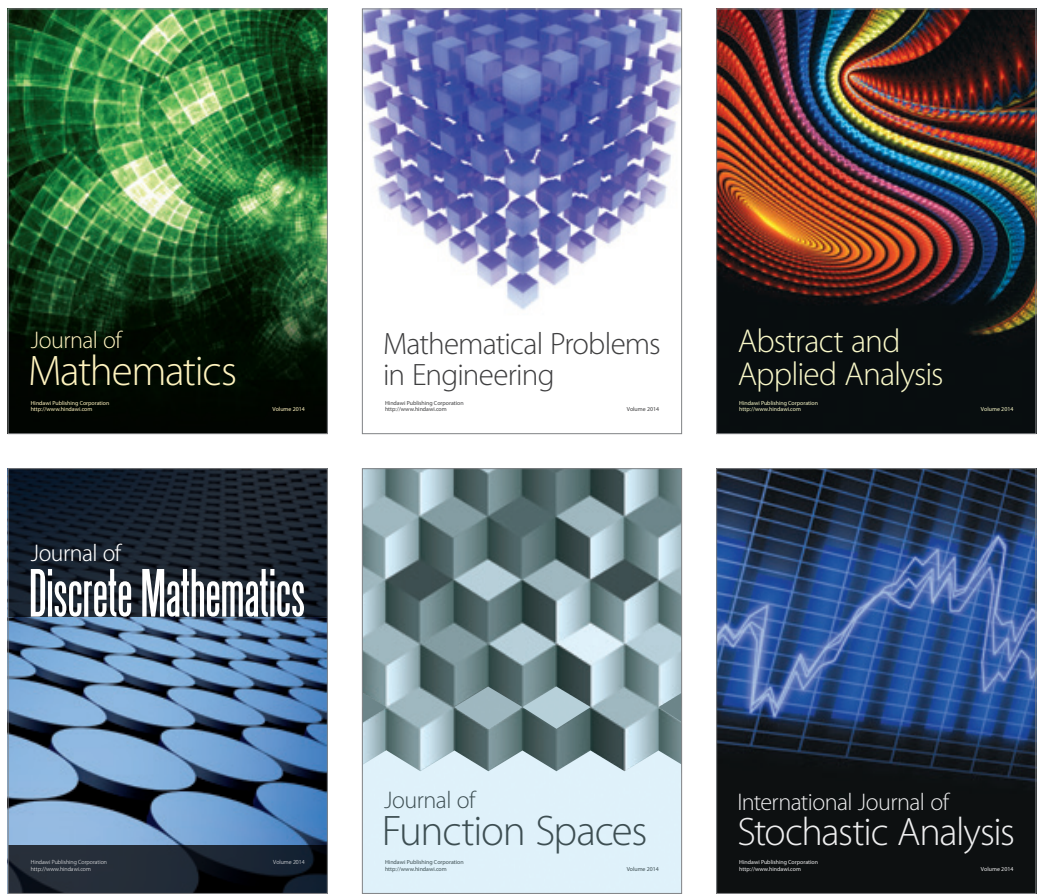

Journal of

Function Spaces

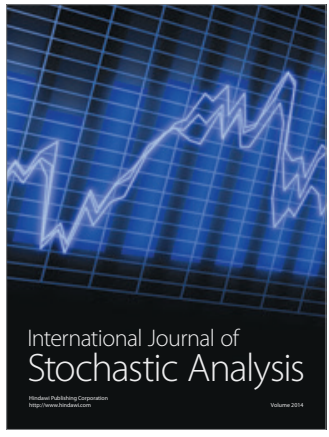

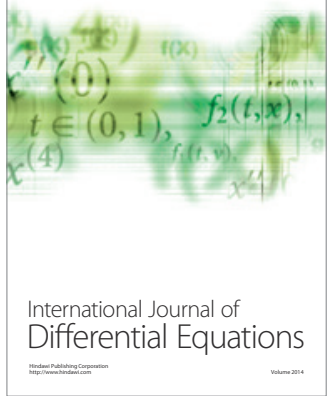
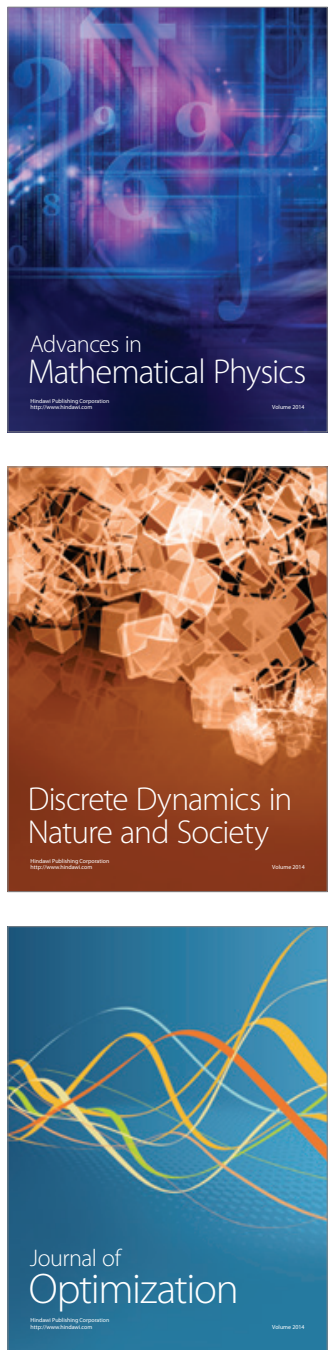